\title{
LA PLACE DU JOURNALISTE DANS LA CONDUITE DU DÉBAT DÉMOCRATIQUE
}

\author{
Jean-Marie Charon ${ }^{1}$
}

Est-il possible d'être journaliste et membre d'un parti politique, voire même élu ? Quel peut être la crédibilité d'un journaliste qui afficherait des options partisanes? Ces questions reviennent fréquemment dans la bouche des élèves des écoles préparant à la profession, le plus souvent lors du traitement de sujets ayant trait à l'éthique ou à la déontologie. Ce sont d'ailleurs ces mêmes questions qui parcourent les réunions publiques, les débats concernant l'information et les médias ${ }^{2}$. Pour le citoyen, comme pour le candidat journaliste poser la question c'est y répondre : il est impensable d'imaginer que le politique, matière noble par excellence, en matière d'information en démocratie, ne suppose pas le même recul, la même indépendance à l'égard des sources que tous les autres types de contenus, qu'il s'agisse de justice, d'économie, d'éducation ou de sciences.

1 Chercheur en sociologie des médias au CNRS.

2 Selon une observation faite par l'auteur, notamment lors d'une mission confiée par la ministre de la Culture et de la Communication, qui devait se conclure par un rapport intitulé : "Réflexions et propositions sur la déontologie de l'information" de juillet 1999, ainsi qu'au cours d'une démarche de débats publics sur le traitement de l'information, dans le cadre des Entretiens de l'information, lancés en 2001 à Hourtin (Gironde). 
Et pourtant la presse française est le produit d'une histoire de plus de deux siècles, au cours de laquelle presse, journalisme et information se sont conjugués avec opinion, engagement, polémique. Pierre Retat ${ }^{1}$ devait montrer qu'en devenant pluraliste la presse de la Révolution française se préoccupait moins d'informations et de faits que d'idées et de choc des opinions. Thomas Ferenczi ${ }^{2}$, comme Christian Delporte ${ }^{3}$ rappelèrent que le journalisme en tant que profession émergea, durant la IIIe République en France, dans un contexte où les carrières politiques et journalistiques s'entrecroisaient, voire se recouvraient, avec des exemples aussi fameux que ceux de Jaurès, Blum ou Clemenceau. Il n'y a qu'un peu moins de soixante ans, la presse française fut reconstruite, au travers des "ordonnances de 44" sur les seuls critères politiques. Comment dès lors rendre compte d'un véritable renversement des points de vue ? Quelles sont les transformations dans la société, comme dans les médias, qui permettent de l'expliquer? Quelle est la place qui est désormais dévolue aux journalistes dans le débat démocratique? L'attente, voire l'exigence de distance entre le journalisme et l'opinion, le journalisme et l'action, de la part des citoyens implique-t-elle une condamnation de toute forme de controverse dans laquelle s'engagerait la profession? Ne faut-il pas voir un signe dans le fait que la polémique se voit bien souvent traitée, à la télévision, comme une forme de spectacle dans laquelle s'illustrent des animateurs dans des programmes ${ }^{4}$, placés désormais hors des espaces d'information proprement dit (reality show, talk show, etc. $)^{5}$ ? Ne doit-on pas s'interroger sur plusieurs formes de "déplacements" qui voient dans le journalisme dit d'investigation ou le "journalisme public", une partie de la profession se revendiquer d'un nouveau rôle au sein du débat démocratique, "au côté de la société"6 ?

1 La révolution du journal (1788-1794) Paris, Éditions du CNRS, 1989.

2 L'invention du journalisme en France, naissance de la presse moderne à la fin du XIXème siècle, Paris, Plon, 1993.

${ }^{3}$ Les journalistes en France (1880 - 1950). Naissance et construction d'une profession, Paris, Éd. Du Seuil, 1999.

4 Tels Thierry Ardisson, Marc-Olivier Fogiel, Jean-Luc Delarue, etc.

5 Cf. le numéro de Réseaux, dirigé par Erik Neveu: "La politique saisie par le divertissement", $n^{\circ} 118$, Paris, Éd. Hermès, 2003.

6 Selon l'expression de journalistes d'investigation, reprise par Jean-Marie Charon dans : Un secret si bien violé - La loi, le juge et le journaliste, Paris, Éd. du Seuil, 2000. 


\section{Les éléments du renversement}

Observateurs des médias et journalistes seniors s'accordent sur un constat, les médias modernes ont perdu le sens du débat et de la polémique politique. L'affrontement des idées, comme des programmes n'y trouvent plus qu'une place limitée dont ils se font au mieux l'écho, mais dont ils ne se sentent plus les acteurs. Les "anciens" se sentaient investis d'une demande de sens, d'engagement de la part de leur public. Ils ne trouvent plus que des traitements distanciés, équilibrés, asceptisés, dans lesquels les questions en débat sont renvoyées aux responsables de partis, de syndicats, de mouvements confessionnels ou d'écoles philosophiques. De tels traitements se voient largement relativisés, au profit d'approches mettant davantage en valeur les questions de personnes (voire même de traitements quasiment people), l'analyse de dossiers souvent très techniques et bien souvent extrêmement complexes, les considérations relatives à l'état de l'opinion avec le recours incessant à toutes formes de sondages. Mettre en évidence les facteurs d'une telle évolution n'est pas aisé. Il est possible au moins d'en évoquer quelques-uns concernant le contexte français de la seconde moitié du $\mathrm{XX}^{\mathrm{e}}$ siècle.

La place qu'occupait l'affrontement des opinions parmi les journalistes ne peut en France s'interpréter sans prendre en compte la nature des institutions politiques qui ont caractérisé la $\mathrm{III}^{\mathrm{e}}$, puis la $\mathrm{IV}^{\mathrm{e}}$ République. L'instabilité des majorités et des gouvernements y était très grande. Les journaux participaient totalement de l'affrontement des points de vues et des nécessaires recherches de combinaisons conduisant à l'instauration des majorités, aussi éphémères fussentelles. L'instauration de la Ve République basée sur des institutions et des majorités beaucoup plus stables, permettant à un personnel politique beaucoup plus gestionnaire de s'installer pour des périodes beaucoup plus longues, conduisit à la montée d'un traitement beaucoup plus technique et explicatif des questions politiques. La question des capacités et des compétences des dirigeants s'installa progressivement aux dépens des idées et programmes proprement dits. L'expérience des alternances, à partir de 1981, devait conforter la représentation selon laquelle la maîtrise des dossiers et la capacité à trouver des solutions pragmatiques avait plus d'importance que les idées proprement dites. 
Ces idées et références idéologiques se trouvèrent encore davantage reléguées au second rang avec la fin de l'affrontement des blocs Est - Ouest et la crise des idéologies. Désormais les repères, les grilles de lectures auxquels se rattachaient les journalistes firent défaut. Il fallait désormais juger sur pièce, dossier par dossier : chômage, éducation, environnement, insécurité, etc. La prégnance de références issues de la vulgate gestionnaire et économique devait conduire à ce que d'aucuns dénonceront comme une forme de consensus mou, avec la fameuse question de la "pensée unique". Non seulement la controverse et le débat marquaient le pas, mais les journalistes perdaient toute autonomie de pensée ${ }^{1}$.

La place prise par la télévision n'est pas non plus à négliger. A la peine dans l'analyse des idées et des projets, le média devenu dominant s'engouffra dans la mise en valeur des personnes et de leurs faits et gestes, sans parler de leurs "petites phrases". Devenue commerciale et concurrentielle, elle s'obnubila de sa capacité à conquérir et préserver des audiences qui ne passaient plus par le spectacle répétitif des face à face de dirigeants politiques. Ces derniers de leur côté n'eurent de cesse de maîtriser une communication qui entendait éviter les moments de confrontation directe entre les champions de partis dont l'avenir pouvait basculer en quelques minutes, faute d'avoir paru suffisamment assuré ou convaincant face à leur compétiteur ${ }^{2}$. Sans doute plus fondamentalement la figure même du journaliste, sous la forme du présentateur, place comme central un rôle "d'accompagnateur du public"3, de "médiateur", sur les lieux et dans l'approche des grands dossiers. Cet accompagnateur, proche de son public, ne saurait être partisan. Il ne peut que cultiver une posture d'objectivité4. Les éléments de polémiques ou de débats, ne sont appréhendés qu'au travers d'une relative mise à distance, dans laquelle le journaliste ne saurait être partie prenante. Quelque part la

1 Dénoncée par exemple dans l'essai de Serge Alimi, Les nouveaux chiens de garde, Paris, Liber, ainsi que les différentes approches du Monde diplomatique, que l'on retrouve également dans le petit livre de François Ruffin, Les petits soldats $d u$ journalisme. Paris, Les Arènes, 2003.

2 Tel Laurent Fabius lors de son face à face avec Jacques Chirac, au milieu des années quatre-vingt, précédant de peu l'arrivée du second au poste de Premier Ministre de la première cohabitation.

3 Selon la formule que nous utilisions dans Cartes de presse, Paris, Stock, 1994, pour décrire le journalisme.

4 Se reporter à ce sujet à l'ouvrage d'Arnaud Mercier, Le journal télévisé, Paris, Presses de Sciences Po, 1996. 
polémique est en quelque sorte tenue à distance sous la forme de l'affrontement de petites phrases contradictoires (dans les journaux télévisés), voire dans la transformation des dernières émissions politiques en des espaces d'affrontements multiples entre des personnes nombreuses (et à statut divers) ne peuvent que polémiquer, sur la forme, la personnalité, le style de chacun, plus que sur le fond.

Il faut enfin évoquer la montée de la question du professionnalisme des journalistes au moment où devait s'élargir très sensiblement l'éventail des domaines couverts par l'information. Désormais les questions de compétence et de maîtrise de la complexité des domaines traités en économie, en sciences, en éducation, etc. vont prendre le pas sur celle des convictions ou des options personnelles des journalistes. La question de la capacité des journalistes à expliquer et rendre compréhensible le monde prend plus d'importance que celle des valeurs dans lesquelles ceux-ci se reconnaissent. Il faut également évoquer le mouvement constant en faveur d'une thématisation des contenus (en presse magazines) sans parler de la segmentation des publics visés qui paraît totalement diluer les anciens clivages basés sur l'ancrage politique des anciennes lignes éditoriales.

\section{Nouvelles attentes du public et dévaluation de la controverse}

Il n'est pas possible d'interpréter le lent déclin des controverses journalistiques en matière d'information politique sans interroger ce que sont aujourd'hui les attentes du public à l'égard des journalistes. Faute de disposer d'études répondant exactement à cette question, il est au moins possible de se référer à trois types de sources qui semblent largement converger sur le sujet : la première est constituée de sondages, dont le principal est celui que devait lancer le quotidien La Croix, la revue Médiaspouvoirs et l'institut SOFRES', qualifié

1 Ces baromètres annuels organisés depuis 1987, avec le partenariat successif de plusieurs magazines (Télérama et Le Point) comportent un volet concernant les appréciations du publics concernant les sujets d'actualité de l'année (événements politiques tels que la montée du Front National, les campagnes électorales ; mais aussi les "affaires" politico-financières; etc.). Ils sont à rapprocher de sondages plus ponctuels organisés par divers organes de presse dont La Vie ou Libération et les instituts de sondages CSA et BVA également sur les affaires de justice, l'investigation, la crédibilité des médias). Une synthèse de ceux-ci avait été 
communément de baromètre sur "la confiance des français dans leurs médias". La seconde prend la forme de plusieurs contributions émanant d'institutions telles que la Commission Nationale Consultative des Droits de l'Hommel et le Conseil Économique et Social $^{2}$. La troisième est le fruit de diverses démarches associatives ayant pour objet le débat public en matière de traitement de l'information ${ }^{3}$.

L'ensemble des matériaux ainsi disponibles, certes disparates et hétérogènes permet de poser une hypothèse qui serait celle d'une dévaluation, voire dévalorisation de la controverse politique au profit de ce que pourraient être les modes de traitements "adaptés" ou "performants" des problèmes et questions auxquels se trouve confrontée la Cité, la collectivité, le corps social. Une telle hypothèse peut être posée au regard des priorités auxquelles se montrent attachés, et semble-t-il de plus le plus, les publics des médias, les destinataires du travail journalistique, ainsi que des réticences que suscitent des modes de traitements dénoncés comme "inutilement" polémiques, "excessivement" agressifs, voire violents à l'égard des personnes publiques.

\section{Fiable plutôt que contradictoire}

En premier lieu semble s'affirmer un point de vue selon lequel le traitement d'une question ou d'un problème ne progresserait que très

proposée dans notre rapport à la ministre de la Culture et de la Communication en 1999, intitulé : "Réflexions et propositions sur la déontologie de l'information".

1 Qui a publié le 21 mars 1995 un avis intitulé "La liberté de la presse et la responsabilité des journalistes - Pour une déontologie de la liberté d'expression".

2 Le Conseil Économique et Social, national, a publié en 1999 un rapport intitulé Liberté d'information et protection du citoyen face au développement des médias, Éditions des journaux officiels. Le Conseil Économique et Social de la région Languedoc Roussillon devait également adopter un rapport sur le même sujet.

3 Figure dans ce cadre l'action menée par l'association Reporters sans Frontières, durant les années 89 - 92 (débats à propos du traitement de la crise roumaine, de la guerre du Golfe, de l'éthique de l'information, ayant donné lieu à publication d'ouvrages édités par l'association). Il faut également prendre en compte durant les années 90 l'initiative de nombre de Clubs de la presse (notamment à Toulouse, Montpellier, Strasbourg, etc.), de même que celle des débats sur le sujet de l'éthique journalistique organisés dans le cadre de l'Université d'été de la communication, par le Crépac, à Hourtin (Gironde). Enfin depuis trois années la démarche initiée par les Entretiens de l'information (cf. Les Cahiers du Journalisme, Lille, avril 2003). 
peu, voire pas du tout, par la simple exposition, reprise, mise en scène des points de vues des acteurs "institutionnels", voire traditionnels, de la controverse publique : gouvernement, dirigeants de partis, responsables syndicaux, etc. De ce point de vue d'ailleurs un déplacement net paraît s'être opéré qui accorderait moins de valeur à des positionnements, des projets, des problématiques d'ensemble ou globales, qu'à l'identification de ce que sont les vrais problèmes, les questions réellement pertinentes, ainsi qu'à la capacité de les résoudre, les traiter de manière satisfaisante.

L'important en matière d'information ne serait plus d'exposer les divergences ou différences de points de vue par la mise en scène des oppositions partisanes, syndicales, idéologiques. Il ne serait pas non plus acceptable que le journaliste ou le média adopte l'un des points de vue, au motif d'opinion, de valeur, de soutien à des personnes, partis, philosophie. D'où la fameuse question redondante dans les sondages sur "l'indépendance" et la dénonciation récurrente du manque d'indépendance (baromètre La Croix - SOFRES, sondage Libération - BVA, etc., sans parler de l'interpellation des médiateurs de France 2, France 3, Radio France, du Monde, quant à une présentation non équilibrée des conflits sociaux du printemps 2003 ou du conflit du Moyen Orient ${ }^{1}$ ). Ne faut-il pas voir une prise en compte de cette exigence du public, dans la manière qu'adoptent désormais les quotidiens pour mettre en scène l'opinion, la prise de position, sous la forme d'expressions d'intellectuels, de personnalités, de spécialistes, d'acteurs du politique ou de la société, dans des rubriques -spécifiques et bien balisées- telles que "Forum" (La Croix), "Horizons - Débats" (Le Monde), "Rebonds" (Libération), etc. Dans ce dernier titre des journalistes peuvent d'ailleurs trouver leur place dans cet espace, à part, marquant encore davantage les moments, habituels, de leur traitement "professionnel" dans le journal et des moments, plus rares, d'expression de points de vue dans les "Rebonds".

L'attente, voire l'exigence, est ici que le journaliste -le médiasoit capable d'adopter un point de vue "compétent", fournissant les éléments pertinents, les analyses, les argumentations susceptibles de

1 On pourra se reporter par exemple aux actes du débat sur "Le traitement du conflit au Moyen Orient", in "Les Entretiens de l'information", Les Cahiers du journalisme, hors série, Lille, avril 2003, pp. 69 à 141. Voir également les différentes Lettres du médiateur de la rédaction de France 2 en 2001, 2002 et 2003. 
résoudre le problème posé. Un point de vue "compétent" est sensé permettre une compréhension des questions et des enjeux afin que le public - citoyen soit en mesure de se faire son opinion et d'agir pour son propre compte. Il est remarquable à cet égard que la nature des problèmes s'est également déplacée, débordant largement celle qui concernerait la seule action politique. Elle prend désormais en compte le comportement de l'acteur économique (dirigeant d'entreprise, cadre, salarié, sans parler de l'actionnaire, y compris le "petit actionnaire" face aux grands groupes multinationaux ${ }^{1}$ ), de l'habitant d'une grande métropole confronté aux problèmes de la banlieue, du parent, investi du devoir de soigner, éduquer, orienter ses enfants, du consommateur, du justiciable, etc. Tous domaines dans lesquels les conséquences des choix et des éventuels erreurs, faute d'être correctement "informé" ou conseillé par les médias, peuvent avoir des conséquences directes, graves et profondes sur la vie ou l'existence de chaque destinataire de l'information.

Il faut probablement voir dans cette représentation du rôle contemporain des médias et du travail journalistique la montée du thème de la "fiabilité de l'information"2, ainsi que de la responsabilité ou de l'irresponsabilité des journalistes. Une responsabilité au sens de "devoir"3 des journalistes à l'égard de leur public et plus globalement de la collectivité. Fiabilité et responsabilité prennent alors beaucoup plus d'importance que la question du positionnement ou de l'argumentation dans le cadre d'une controverse publique. Il peut alors paraître significatif de voir émerger des démarches associatives dont l'ambition serait de dégager les critères professionnels permettant d'accéder à une telle fiabilitét .

\section{Le rejet des excès de la polémique}

Il faut également s'interroger sur une forme de mise à l'écart de la controverse dans le mode de traitement des problèmes, sur le fait de

1 Cf. la place prise par les associations, groupements de ces petits actionnaires face au management de groupes tels que Vivendi ou France Télécom.

2 Cf. à ce propos le chapitre 9: "Le débat sur la crédibilité des médias", dans notre ouvrage, Les médias en France, Paris, La Découverte, 2003.

3 Selon l'acception d'Alain Etchegoyen, dans La wraie morale se moque de la morale - Etre responsable, Paris, ,Ed. du Seuil, 1999.

4 Telle l'association "AQUIT" créée par Jean Robin, en 2003. 
savoir si elle ne tiendrait pas pour une part aux formes même de l'expression, des attitudes, des formulations des argumentaires contradictoires qui seraient perçus comme déqualifiants, voire inacceptables parce que participant d'une atteinte aux personnes, à leur réputation, au respect qui leur serait dû. Une sorte "d'atteinte aux bonnes mœurs démocratiques" pourrait-on dire. Le rappel dans une assemblée publique des qualificatifs, des invectives, des termes utilisés dans les polémiques qui agitaient la presse française durant l'entre-deux-guerres, notamment à l'égard d'une personnalité tel que Léon Blum, semble, à chaque fois, glacer d'effroi ou du moins provoquer un profond malaise de l'auditoire. Comment pouvait-on recourir à de telles formes de débats par presse interposée, entre éditorialistes, dans le cadre d'une société démocratique ? Tous les sondages récents sont à cet égard convergents, du moins en France, l'atteinte aux personnes privées, aux individus, voire aux dépositaires de fonctions est très majoritairement condamnée par l'opinion publique. Il est à cet égard tout à fait significatif d'observer que dans un même sondage concernant les relations médias - justice, à propos des affaires et du journalisme d'investigation, celui-ci se voit crédité d'un rôle positif dans l'affermissement des pratiques et institutions de la démocratie, alors qu'un peu plus loin il sera regretté que cette même investigation porte atteinte aux personnes et à leur réputation ${ }^{1}$.

La controverse, au moins dans sa forme polémique, se heurterait à une réticence de l'opinion qui, a priori, rejette régulièrement, sondage après sondage, les atteintes aux personnes, le non respect de la présomption d'innocence, les modes de traitements qui relèveraient d'une certaine forme de violence ou d'acharnement. La réaction d'une partie du public à l'égard du traitement du Front National, entre les deux tours de l'élection présidentielle de 2002 illustre parfaitement le phénomène. Autant l'expression publique du rejet de l'extrême droite s'exprima avec force dans la rue, les médias et notamment les télévisions, TF1 et France 2 se voyant reprocher d'avoir fait le lit de JeanMarie Le Pen ${ }^{2}$, autant le public réagit négativement dans le courrier des lecteurs, les adresses aux médiateurs, face à un ton de commentaire, un mode d'interview des leaders du Front National, qui fut jugé

1 Dans le sondage La Croix-Télérama-SOFRES de 1998.

2 Les banderoles des manifestations affirmaient ainsi : "Médias coupables!", "Médias responsables !", alors que la formule du responsable socialiste, Julien Dray, "TFHaîne", faisait florès, en fleurissant sur les murs des banlieues. 
partisan, inadapté, voire condamnable du point de vue du professionnalisme attendu des journaliste ${ }^{1}$.

\section{L'impact de l'encadrement législatif et institutionnel du traitement de l'information}

Peut-être faudrait-il s'interroger en quoi l'évolution du droit de l'information, voire la pratique de l'instance de régulation de l'audiovisuel, soit le CSA depuis 1989, ont pu contribuer à faire régresser les espaces de la controverse journalistique et surtout ses formes les plus exubérantes. L'évolution du droit, de son interprétation par les tribunaux, de sa mise en œuvre par les acteurs du débat politique, sans parler des recommandations successives du CSA ne sauraient, bien entendu, s'interpréter sans tenir compte des attentes et représentations du corps social, évoquées plus haut. Il est en tout cas tout à fait évident que si l'on devait mettre en regard les formes prises par la controverse politique de l'entre-deux-guerres, celle-ci butterait immédiatement sur la pratique contemporaine des plaintes pour diffamation ${ }^{2}$, sans parler du texte de 1993 sur les propos racistes, voire sur la présomption d'innocence de 1993 et $2000^{3}$. Bien que les formes de débats dans les médias soient devenus beaucoup plus sages, courtoises et modérées, il faut remarquer que les procédures pour diffamation sont devenues extrêmement fréquentes. Même s'il faut reconnaître qu'elles concernent plus souvent aujourd'hui les questions liées aux "affaires" que les controverses politiques proprement dites.

L'influence des recommandations du CSA s'exprime principalement aujourd'hui sur les questions liées au respect du pluralisme et à la manière extrêmement "comptable" dont celui-ci est traité, surtout dans les périodes électorales. Dans un contexte, certes un peu particulier, tel que celui de la campagne présidentielle de 2002, avec de très nombreux candidats (16), il devait conduire mécaniquement à rejeter un traitement sous forme de face à face, ou de duels entre les princi-

1 Ce point de vue transparaît notamment du débat organisé sur "Le traitement de la campagne électorale" lors des Entretiens de l'information d'août 2002. Se reporter à son compte rendu dans le hors série des Cahiers du journalisme déjà cité (pp. 229 à 274).

2 Cf. par exemple, Emmanuel Derieux, Droit des médias, Paris, Dalloz, 1995.

3 Cf. Jean-Marie Charon, Les médias en France, Paris, La Découverte, 2003, le chapitre II : "La législation de l'information et des médias", pp. 23 à 35. 
paux leaders, au profit d'un suivi - compte rendu de la campagne de chacun des candidats.

Élimination des formes les plus rugueuses de la controverse, contraintes propres à certains moments forts du débat politique, sans être totalement sans effet, il faut reconnaître que l'intervention du droit ne saurait rendre compte, à elle seule, du reflux de la controverse dans le traitement du politique, qui renvoie davantage aux transformations profondes du journalisme politique, comme à la relation entre les médias et leur public, dans les dernières décennies. Tout se passe comme si progressivement les espaces de la polémique, y compris politique s'étaient déplacés glissant par exemple de la presse écrite vers le livre, ou des plages d'information vers les "programmes", notamment avec "l'infotainment" en télévision' ou en radio, les animateurs ou humoristes prenant la relève des journalistes, avec par exemple le phénomène des "Guignols de l'info" sur Canal + , ou des différents formats d'émissions animées par des animateurs - polémistes tels que Laurent Ruquier, Thierry Ardisson ou Marc Olivier Fogiel $^{2}$. Une autre forme de glissement, qui elle concerne les journalistes, est celle qui met en scène les spécialistes de l'investigation, avec le développement des "affaires" à partir du milieu des années quatre-vingt.

\section{Journalisme d'investigation et contrôle des pouvoirs au nom de la société}

La seconde moitié des années quatre-vingt voit émerger un phénomène nouveau dans la presse française, celui des affaires "politico-financières", fruit du développement d'une forme de journalisme d'enquête qui se veut particulière et se qualifie elle-même de

1 Cf. "Aurélien Le Foulgoc : 1990-2002 : une décennie de politique à la télévision française", Erik Neveu : "De l'art (et du goût) d'éviter la politique. La démocratie du talk-show version française (Ardisson, Drucker, Fogiel)", Marie Lhérault, Erik Neveu: "Quelques dispositifs de talk-shows français (1998-2003), Réseaux, $n^{\circ} 118,2003$.

2 Sans parler de la polémique de l'automne 2003, à propos du projet d'émission de TF1, "36 heures", dite de "politique réalité" qui aurait consisté à filmer un homme politique, immergé 36 heures dans le quotidien d'un foyer français. Ici la controverse semble devoir s'arrêter à l'opposition entre les partisans et les adversaires d'un programme de type. 
"journalisme d'investigation". Au-delà de la nature des premières "affaires" qui vont sortir dans des journaux comme Le Monde ou Libération, et des hebdomadaires comme L'Express, le Point, Le Nouvel Observateur et bien évidemment Le Canard Enchaîné, le choix du qualificatif de "journalisme d'investigation" par des journalistes tels qu'Edwy Plenel' ${ }^{1}$, Jean-Marie Pontaut ${ }^{2}$, Gilles Gaetner et bien sûr l'un de leurs précurseurs Jacques Derogy, est signifiant, parce qu'il s'inscrit délibérément et consciemment dans une référence au journalisme d'investigation nord-américain et tout particulièrement le symbole du Watergate. Mark Hunter ${ }^{3}$, dans sa thèse, puis dans son ouvrage sur le journalisme d'investigation, met bien en évidence le phénomène, et tout particulièrement sa parenté symbolique, même si les pratiques et les contextes français et nord-américains sont, bien sûr, très différents.

Ce journalisme d'investigation d'abord souvent mené par quelques individus, anciens militants politiques (tels Jacques Derogy ou Gilles Perrault, voire Edwy Plenel ${ }^{4}$ ) va se trouver progressivement le fait de journalistes qui n'ont pas de parenté avec le journalisme politique et qui se vivent comme en rupture ou en position critique à l'égard du politique. Il ne s'agit plus de rendre compte ou de se situer dans le débat politique, mais de se placer dans un point de vue, en recul, critique, à l'égard des institutions politiques et gouvernementales (police, justice, partis, gouvernement, présidence de la République). Le point de touche principal et initial étant celui de

1 L'actuel directeur de la rédaction du Monde, dont la première grande affaire sera celle des "Irlandais de Vincennes", revendique très tôt ce qualificatif de journalisme d'investigation, par exemple dans le numéro de la revue Esprit de décembre 1990, intitulé "Où va le journalisme ?", ainsi que dans ses ouvrages $L a$ part d'ombre, Paris, Stock, 1992, puis Un temps de chien, Paris, Stock, 1994, etc.

2 Comme le marque bien le titre de son ouvrage commun avec Jacques Derogy: Investigation passion - Enquête sur 30 ans d'affaires Paris, Fayard, 1993, alors que leurs précédents ouvrages titraient sur le terme d'enquête, tel Enquête sur trois secrets d'Etat, Paris Robert Laffont, 1986 ou Enquête sur un Carrefour dangereux, Paris, Fayard, 1987.

3 Aspects du journalisme d'imvestigation : psychologie, méthodologie et stratégies narratives de trois enquêtes européennes, incluant une histoire comparative de l'investigation en France et aux Etats unis depuis 1973, sa thèse soutenue à l'Université de Paris II - Panthéon Assas, 1995, ainsi que Le journalisme d'investigation, Paris, PUF, coll. "Que sais-je ?", 1997.

4 Les premiers n'ont jamais fait mystère d'un engagement initial au sein du Parti Communiste Français, alors que le dernier a expliqué dans l'un de ses derniers ouvrages, Secrets de jeunesse, Paris, Stock, 2001, son itinéraire de militant trotskiste, au sein de la Ligue Communiste Révolutionnaire. 
l'indépendance de la justice et des pressions du politique à l'égard des juges d'instruction et des parquets, autrement dit des tentatives d'étouffement d'affaires qui mettent en cause la gendarmerie (les Irlandais de Vincennes), les services secrets (le Rainbow Warrior), le financement des partis (Carrefour du Développement, Urba, salaires fictifs de la mairie de Paris, etc.), l'entourage du Président de la République (Péchiney Triangle, Elf), le trucage de marchés publics (office des HLM de Paris), le Président lui-même (Écoutes de l'Élysée), la santé publique (sang contaminé, ARC), etc. Progressivement il va soulever la question de la corruption des politiques et des hautes sphères politiques (Elf - Dumas, MNEF, etc.), illustrée par la condamnation d'un ministre tel que Alain Carignon (pots de vins payés par une compagnie d'eau à l'ancien maire de Grenoble) ou de l'ancien maire de Lyon, Michel Noir, etc.

Le moment où le journalisme d'investigation prend son essor (fin des années 80 ) puis va culminer (les années $2000-2001)^{1}$ est assez signifiant, dans la mesure où il coïncide, d'une part avec la fin de l'affrontement entre les blocs, avec la dilution des repères politiques et idéologiques qui l'accompagne, au niveau international et d'autre part la succession des périodes dites de cohabitation en France (85$88,93-95,97-2002$ ). Le journalisme politique y a perdu largement ses marques. Il n'est pas exagéré de parler de crise de celui-ci, marquée dans nombre de rédactions (dont celle du Monde, un moment de Libération ou au Nouvel Observateur) par l'arrivée aux postes de direction ou du moins d'encadrement, de journalistes d'investigation. Comme si le positionnement critique, en retrait, en revendication d'une posture de contrôle du politique ou de contre-pouvoir, marquait une forme d'alternative à l'égard d'un journalisme politique engagé dans le débat ou spécialiste de l'analyse du débat politique lui-même.

Dans son positionnement explicite le journaliste d'investigation se situe en retrait, voire à l'écart de la controverse politique ellemême : il va simultanément s'attaquer à la pratique d'élus, de ministres ou de partis, de droite comme de gauche, sur des faits similaires tels que les financements occultes de partis, l'abus de bien sociaux, la corruption, etc. En même temps il est l'acteur central d'un déplacement de cette controverse qui va désormais s'établir entre les

1 Se reporter sur ce point à notre ouvrage Un secret si bien violé - La loi, le juge et le journaliste, op. cit., ainsi que notre article dans Hermès, $n^{\circ} 35$, "Le journalisme d'investigation et la recherche d'une nouvelle légitimité", Paris, 2003. 
médias eux-mêmes et les personnes, les partis, les institutions, les entreprises qui se trouvent mis en cause par les affaires. Ce faisant la nature de la controverse change puisqu'elle ne porte plus sur des idées, des programmes, des analyses, mais sur des faits, des arguments de droit, le respect de procédures, voire de principes moraux. Les protagonistes de la controverse sont des médias -et même des journalistes en tant qu'individus, menant les investigations et auteurs des révélations- et les personnes mises en cause, leurs avocats, sans oublier l'intervention de personnes, d'associations (de victimes du sang contaminé par exemple), ainsi que leurs avocats. La controverse se mène, bien sûr, au travers de prises de positions publiques, de débats publics devant les tribunaux, mais surtout aussi par médias interposés (Le Président du Conseil Constitutionnel, ancien ministre des Affaires étrangères, Roland Dumas, répond au Monde dans une interview dans le Figaro, par exemple).

L'engagement, puis le développement de la controverse par les médias et dans les médias, n'a rien de fortuit ou de marginal, puisque celle-ci est conçue par les journalistes d'investigation eux-mêmes comme constitutive du développement de l'affaire. Edwy Plenel expliquera ainsi à de nombreuses reprises comment le choix du moment et des termes du premier article traitant d'une affaire vise à faire bouger et réagir, souvent par médias interposés, les différents protagonistes de celle-ci ${ }^{1}$. L'enjeu et le lieu de la controverse dans les médias, soit dans l'espace public est lui aussi tout à fait signifiant puisqu'il consiste d'abord à mettre en lumière des faits, des actes, des comportements qui se voulaient secrets, cachés. Il exprime également la place et la légitimité que se donne le journaliste dans l'investigation, soit un rôle de contrôle du et des pouvoirs, aux côtés ou au nom du public, de la société, des citoyens ordinaires. Enfin il est possible d'y voir au moins pour partie un mode d'illustration d'un modèle de société démocratique "moderne", largement partagé par ces journa-

1 Cf. les ouvrages d'Edwy Plenel déjà cités, ainsi que l'ouvrage de Pontaut et Derogy. Dans Les journalistes, Paris, Syros, 1994, Jean-François Lacan, observateur, alors journaliste au Monde, décrit bien l'un des moments que va choisir Edwy Plenel pour sortir son premier papier sur l'une des grosses affaires de délit d'initiés, un samedi, attendant en cela le développement de celle-ci au cours du week end, avec la promesse de réactions importantes dès le lundi suivant, dans les médias. 
listes ${ }^{1}$, qui s'appuierait sur un triptyque : transparence, régulation, contrôle.

Dans le déplacement qu'opère le journalisme d'investigation, dans le développement de la controverse, il faut revenir sur deux points qui paraissent majeurs : d'une part le registre des idées, des modes de traitements des problèmes de la Cité par les acteurs du politique, est largement délaissé au profit d'un registre d'argumentation juridique et institutionnel. C'est au moins l'expression apparente qu'il se donne. Il est cependant incompréhensible sans évoquer un soubassement qui lui relève du registre éthique. L'accent mis sur les questions de corruption, de mensonge public, d'irresponsabilité concernant les personnes ou les groupes qui prétendent exercer les fonctions de pouvoir et de représentation publique, paraît en effet totalement indissociable des motivations morales d'une telle forme de journalisme. Cette dimension, voire prétention morale, constitue un point d'achoppement et de controverse, très vif, avec les journalistes politiques, tout comme avec nombre d'acteur politiques, qui contestent la légitimité des journalistes d'investigation dans ce domaine, les renvoyant à des motivations beaucoup moins élevées, telles que des tentations démagogiques ou poujadistes, voire purement mercantiles. C'est par exemple le registre que privilégiera le Président de la République, Jacques Chirac, lorsque dans ses vœux du 31 décembre 2000, il stigmatisera la simple motivation à "vendre du papier". D'autre part le journaliste d'investigation ne situe plus sa démarche du point de vue du débat politique, du bien public ou de la marche de la Cité, il entend intervenir au côté du citoyen. De ce point de vue il pourrait bien rejoindre un mouvement plus général, dans lequel il s'apparenterait à une approche pourtant apparemment assez lointaine qu'est celle du "journalisme public".

\section{Régulation et transparence - le déplacement de la controverse}

La montée du couple régulation - débat public, au moment où l'image du politique et les mécanismes de la décision font problème, parce que lointains et paraissant sans prise possible pour le citoyen, ne

\footnotetext{
I Pour plus de développement se reporter à l'article de Hermès déjà cité, ainsi qu'au chapitre 6 : "Une nouvelle légitimité" de notre ouvrage déjà cité.
} 
constitue-t-elle pas une opportunité pour les médias de se poser comme le lieu où se conduirait le débat public ? Les journalistes dans cette perspective participeraient ainsi d'un rôle d'intermédiaire, de metteur en scène et de facilitateur du débat public. C'est bien sûr ce que suggère la démarche du "journalisme public", telle que la décrit Thierry Watine ${ }^{1}$, par exemple. Des problèmes, principalement locaux, des décisions mettant en cause des intérêts contradictoires, seraient ainsi présentés et mis en débat au travers de leur traitement dans et hors des médias, via l'intervention de journalistes, y compris par l'organisation de débats sur les lieux mêmes où ceux-ci se posent ou pourraient prendre effet. Dans les principes qui fondent le journalisme civique, Thierry Watine en propose deux notamment qui résument bien cette approche: "La presse peut aider les citoyens à régler concrètement certains problèmes plutôt que de les inciter -consciemment ou non- à la passivité ou au désengagement". "Contrairement à leur inclination naturelle à mettre de l'huile sur le feu, les médias doivent davantage contribuer à une amélioration de la qualité et de l'utilité des débats publics". Il faut reconnaître, qu'aujourd'hui, en France une telle approche reste peu développée et se présente plus comme une perspective apte à renouveler une presse locale dont le traitement, dit de proximité, par trop institutionnel et descriptif apparait largement vieilli.

Il est en revanche intéressant de noter la manière dont ces dernières années les radios ont progressivement fait évoluer des émissions de débat dans lesquelles les auditeurs sont amenés à intervenir, tel le "Téléphone sonne de France Inter". Non que le principe de l'intervention à l'antenne via le téléphone, de représentants du public soit tout à fait nouvelle. Mais dans des périodes de crise aiguë, de grand trouble collectif, comme après les attentats du 11 septembre, après l'arrivée du candidat de l'extrême droite au second tour des élections présidentielles de 2002, en France, avant, puis au moment de la guerre en Irak, etc. des éditions spéciales, prolongées de ces "antennes ouvertes" ont vu se développer une forme de débat dans lequel intervenaient des experts, spécialistes des questions posées, des

1 Dans son article dans Hermès $n^{\circ} 35$ : "Le modèle du journalisme public", ainsi que son article plus ancien des Cahiers du journalisme, $\mathrm{n}^{\circ} 1$ : "Le journalisme public aux États-Unis : émergence d'un nouveau concept", Lille, juin 1995. Voir également l'approche qu'en fait Bertrand Labasse dans Une dynamique de l'insignifiance - mes médias, les citoyens et la chose publique dans la "socié té de l'information", Lyon, Presses de l'Enssib, 2002. 
journalistes, mais aussi de nombreux auditeurs. De plus en plus les journalistes-animateurs acceptant et organisant la discussion contradictoire des auditeurs. Les télévisions, bien que moins adaptées, telles France 5 (C dans l'air), LCI, peut-être parce que moins grand public s'y sont, elles aussi, essayées.

\section{Réinvestir et se réapproprier les lieux et les thèmes de débats}

D'un certain point de vue, après avoir pu, durant près de deux décennies, observer qu'une forme de controverse sur des sujets de société extrêmement divers, mais aussi des sujets à contenu politique avait tendance à migrer des rédactions, de l'information, vers les "programmes" sous forme d'émissions de plateau, dites "magazines" (notamment sous la houlette de Jean-Luc Delarue sur France $2^{1}$ ) ou de divers forums, dans lesquels les principes d'expression publique et de représentativité étaient le plus souvent manipulés au profit d'une recherche de spectaculaire, l'heure serait peut-être à ce que les journalistes réinvestissent ces formes de controverse publique. Au regard des émissions d'information débat, de type "Téléphone sonne" le rôle du journaliste a évolué. Il y jouerait d'abord un rôle de médiateur, définissant les thèmes de discussion, les introduisant, organisant les modalités de la prise de parole et de l'échange. Éventuellement des journalistes peuvent y être des acteurs par leur spécialité ou leur point de vue. L'essentiel est cependant qu'il sollicite et accueille la parole de différents spécialistes, acteurs de la société, au même titre que la parole citoyenne. Un phénomène assez semblable serait à l'œuvre dans la presse quotidienne au travers d'espaces tels que les "Horizons - Débats" du Monde, "Forums" de La Croix ou pages "Rebonds" de Libération. Dans ces dernières également les journalistes du quotidien, y interviennent d'ailleurs à leur tour et de plus en plus souvent, mais dans une forme et une présentation, identique à celle d'intellectuels, d'acteurs divers, voire de lecteurs, simples intervenants de cette forme prise par la controverse publique ${ }^{2}$.

1 Notamment "Ca se discute".

2 Dans le numéro du 4 septembre 2003, Jacques Amalric, ancien directeur de la rédaction, publie son point de vue aux côté de ceux d'un "écrivain " et de celui du directeur de l'Espace éthique Assistance publique - Hôpitaux de Paris. Dans celui 10 septembre 2003, par exemple, une lettre du "courrier" de Guy Benoît, 
L'enjeu ne serait-il pourtant pas moins que la controverse ainsi réappropriée par les médias et "gérée", accompagnée, par les journalistes, constituerait une forme de "transfert" des débats publics traditionnels, des meetings publics aux différentes formes de forums locaux, vers les médias eux-mêmes. Il dessinerait en tout cas en pointillé une forme nouvelle du rôle du journaliste dans le débat démocratique. Une forme dans laquelle le journalisme politique, en tant que tel, semble tarder à s'impliquer réellement, comme jaloux de sa compétence, de sa position (de proximité avec les élites politiques) ou de son rôle traditionnel. La crise que connaît pourtant celui-ci ne lui permettra sans doute plus très longtemps de se situer ainsi comme en marge de cette forme de controverse démocratique ainsi redessinée.

“psychiatre honoraire", se situe en vis à vis d'un point de vue d'un Rebonds proposé par un écrivain, aux côté de la chronique de Pierre Marcelle et d'un autre point de vue d'un "professeur en communication"; etc. 